\title{
An Advanced Education Prognostic Miniature Exploitation Random Forest Technique
}

\author{
A. Laxmiprsanna, P. Shirisha
}

\begin{abstract}
The precept goal of the higher instructive affiliation is to offer excessive notable and important education to its understudies. the 2 desires of statistics mining in Indian guidance framework is to have a look at and enhance the account technique facts mining propels development; type out \& speak approximately very last outcomes this is created via the usage of the usage of an information mining technique. This paper centers at some stage in the model portrayal for reading the numerous records mining techniques in Indian guidance framework. moreover the paper surveys a similar research of ID3, $k$-manner, Naïve Bayes, Random wooded vicinity calculation. in this paper, we've got have been given proposed the approach of Random wooded location to foresee the profession choice for the 12th going out understudies. The usage of Random wooded location has helped the understudies to truely receive a proper desire consistent with their advantage and aptitudes and acts a lifelong advertising representative tool package deal.
\end{abstract}

Keywords: Indian schooling device; data mining; Random wooded area

\section{INTRODUCTION}

Training is an organization enterprise or exertion of the senior human beings to unfold their belief to the younger people of society. it's far in this way a foundation, which assumes an critical interest in keeping up the propagation of manner of lifestyles with the useful resource of the use of the use of coordinating a person along alongside together along collectively along with his desired public. in any caseknowledge, in India, the training framework has some real lacunae[3]. in recent times the big problems in the instructive affiliation are, not having increasingly talented, a achievement and unique instructive strategies.these days the huge issues within the instructive affiliation are, not having often effective, a success and particular instructive strategies.

There exist absence of effective and enough studying in Indian instructive framework which hampers the framework the executives to get their dreams. Along those traces, statistics mining is taken into consideration due to the fact the maximum appropriate innovation which gives greater expertise into the modern-day-day truly as instructive regions helping in taking higher alternatives and frightening them to carry out efficiently. Records mining innovation goes approximately as a scaffold some of the lacunas and Indian instructive framework.

Statistics mining method turns on a few data mining tactics at the manner to decorate the adequacy, effectiveness and the exactness of the strategies. for this reason, this development permits in improving the Indian instructive framework by using the usage of the use of growing instructive framework productivity, proscribing understudies drop-out rate, continuously increasing understudies development rate, understudies consistency today's, at the identical time instructive development fee, understudies success, increment in understudies studying price[6]. Along the ones strains, to perform the general great want information within framework encourages chiefs adroitly. Ordinary wooded vicinity is one of the dynamic troupe mastering techniques which reasons the understudies to take right choice for his or her proper career alternatives after board assessments. This facts mining approach trains the understudy with a selected pathway to coordinate his/her more career in a fulfillment way.

\section{TECHNIQUES}

Statistics mining in Indian training framework has a few enlarge defeated the lacunae via brilliant strategies. It is deciding on up prominence due to feasible, efficient and specific toward Indian training framework. The dataset accomplished on this examination includes information of sophistication 10th and twelfth understudies of vocation guiding. The informational index is carried out to enhance the exhibitions, expect, or center spherical abilities of understudies through the use of the usage of numerous order systems.

Determine 1 the entire jogging big records understudies guiding. Vital arrange, facts approximately understudies of sophistication 10th and twelfth had been accumulated and is referred to as as statistics pre-getting ready degree. Within the subsequent diploma, evacuate the vain facts and in reality applicable facts may be sustained to the database. Inside the wake of tending to the understudies statistics, attempted numerous calculations adequate-manner, woodland adequate-manner antique \& commonly broadly utilized calculations applied for grouping larger statistics primarily based databases. Harmless Bayes is one of the measurable classifier techniques which pass about as a speculation for an entire lot of comparing inclinations in a database. This approach acknowledges the adequacy of precise belongings for a given magnificence and its affiliation with different education [2] [5]. The alternative calculation is the Random wooded area, which factors within the maximum crucial randomization through sacking. This technique of the usage of Random woodland aides on dealing with lacking traits and sort symptoms and problems. 


\section{An Advanced Education Prognostic Miniature Exploitation Random Forest Technique}

The third last stage expresses the use of Random backwoods calculation to the preparation informational collection with better yield and the exhibition of every understudy are evaluated [6].

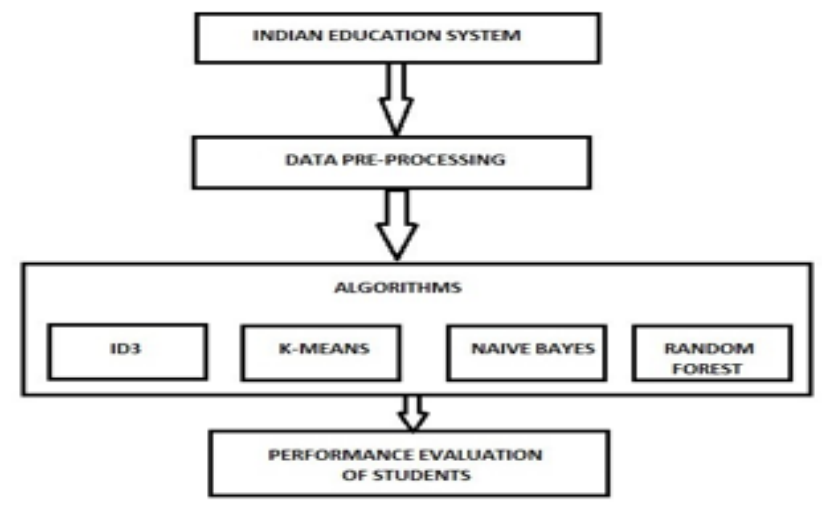

Figure 1. Paradigm of proposed model

The preparation informational collection, appeared in Table: 1 contains detail data of the understudy like Student ID, Gender, and so forth. The entire understudy data detail is utilized as the information dataset.

Table 1. Student related variables

\begin{tabular}{|c|c|}
\hline ATTRIBUTES & VARIABLES \\
\hline Student ID & Student ID \\
\hline Gender & Male/Female \\
\hline Students category & Unreserved/ OBC/ SC/ ST \\
\hline Medium of Teaching & Hindi/English/ Local \\
\hline Stream & Science/ Arts/ Commerce \\
\hline 10th Grade & Excellent/ Average/ Poor \\
\hline 12th Grade & Excellent' Average/ Poor \\
\hline Type of coaching & Online/ offline \\
\hline Scholarship & Yes /No \\
\hline Admission type & $\begin{array}{l}\text { Entrance } \\
\text { exam/Management }\end{array}$ \\
\hline Type of coaching & Yes/No \\
\hline Material & Text book / Online / Both \\
\hline Extra curriculum & $\begin{array}{l}\text { NCC /Scout / Guide / } \\
\text { Sports \& heritage } \\
\text { activities/ both }\end{array}$ \\
\hline Efficiency & Good/Average/Poor \\
\hline Father's occupation & $\begin{array}{l}\text { Service, Business, } \\
\text { Agriculture, Retired, NA }\end{array}$ \\
\hline Mother's occupation & $\begin{array}{l}\text { House-wife (HW), Service, } \\
\text { Retired, NA }\end{array}$ \\
\hline Parental income status & High Medium/ Low \\
\hline
\end{tabular}

\section{RANDOM FOREST}

The Random timberland idea was first presented by Tin Kam Ho. Irregular timberlands or arbitrary choice woodland is a learning procedure for grouping and relapse. It is utilized in the development of choice trees at preparing time and gives yield classes that is as the order classes or mean expectation (relapse) of the individual tree[1].
Basic Random forest Algorithm:

ConsiderNstudent be the no. of students to create for each of $\mathrm{N}$ - students iterations. Where mtry is no. of predictors to try at each split.

$>$ Choose another bootstrap test from the preparation set.

$>$ Develop an un-pruned tree on this bootstrap.

> Arbitrarily, pick Mtry indicators and locate the best part utilizing just these indicators at each inward hub

Each Nstudentprompts the biggest degree conceivable with no pruning.

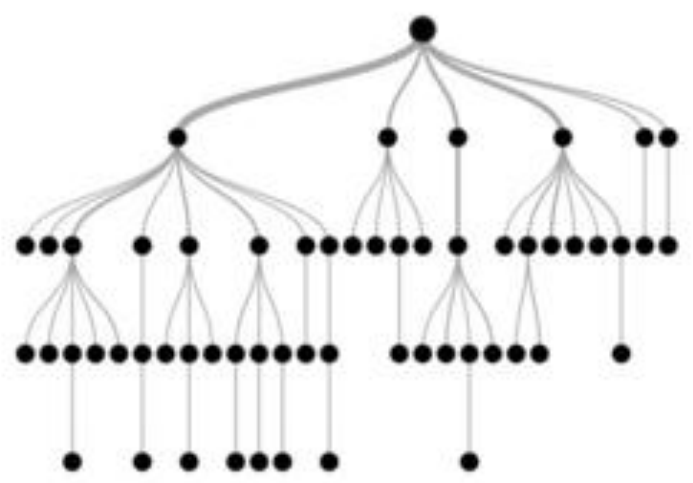

IV. RESULTS \&DISCUSSION

Test, examples thought about. demonstrates precision as far as rate for various classifiers with the expanding informational index foresee adjustment conduct, woodland strategy is utilized understudy procedure recognizes moderate student and sharp student, recoup the disappointment at the earliest opportunity, makes suitable move to improve the poor area understudies in a right way. The examination of understudy's execution utilizing classifier calculations like choice tree grouping, choice woods result reasoned informational index continues expanding, Random backwoods gives better outcome or exactness.

\begin{tabular}{|c|c|c|c|c|}
\hline \multicolumn{5}{|c|}{ Table 2: Prediction accuracy } \\
\hline \multirow{2}{*}{$\begin{array}{l}\text { Dataset } \\
\text { size }\end{array}$} & \multicolumn{4}{|c|}{ Accuracy (\%) } \\
\cline { 2 - 5 } & ID3 & K-means & $\begin{array}{l}\text { Nanre } \\
\text { Byes }\end{array}$ & $\begin{array}{l}\text { Random } \\
\text { forest }\end{array}$ \\
\hline 20 & 62 & 40 & 40 & 60 \\
\hline 80 & 64 & 55 & 62 & 78 \\
\hline 160 & 72 & 43 & 81 & 79 \\
\hline 200 & 75 & 54 & 59 & 80 \\
\hline
\end{tabular}




\section{CONCLUSION}

This paper information a excessive degree for the understudies to select for the extra promising time to include specific and precise exam. due to the reality the productivity, precision, and adequacy expect the important pastime at some level within the time spent Indian education framework, utilization of the Random wooded area method gives us a truly quality answer for this present reality understudy's training. In this paper, we've were given utilized the method woodland foresee choice 12th going understudies. The wooded area understudies honestly delivery of right suitable desire in line with their gain and aptitudes. The final objective is to provide an advanced realize-the way to structure a superior Indian training framework for Indian understudies with the success end stop result. This audit may also moreover advantage out to larger highlights to understand complicated desire databases in a powerful manner.

\section{REFERENCES}

1. Rao, K. Prasada, MVP. Chandra Sekhara, and B. Ramesh "Predicting Learning Behavior of scholars exploitationClassification Techniques." International Journal of laptop Applications, Volume 139, Issues 7, pp: 0975 - 8887, April 2016.P.Veeramuthu "Analysis of Student Result Using Clustering Techniques" International Journal of Computer Science and Information Technologies, Volume 5, Issues 4, pp: $5092-$ $5094,2014$.

2. P.Veeramuthu "Analysis of Student Result exploitation agglomeration Techniques" International Journal of technology anddata Technologies, Volume 5, Issues 4, pp: 5092 5094, 2014.

3. Goyal, Monika, and RajanVohra "Applications ofknowledgemining in educational activity." International journal of technology, Volume 9, Issues 2, pp: 113, March 2012. 